\title{
Acerca de cómo los aymara aprendieron el castellano (terminando por olvidar el aymara)
}

HANS GUNDERMANN ${ }^{1}$

\section{RESUMEN}

¿Cómo es que entre la población indígena de Arica y Tarapacá durante el poco más de un siglo y medio de vida republicana, el aymara ha transitado desde el estatus de lengua dominante a la de lengua subordinada en rápido retroceso ante el castellano? Se diagnostica la situación actual de esta lengua y se desarrolla una explicación histórica de los principales cambios. Una y otra se apoyan en una encuesta sociolingüística y en información etnográfica e histórica. Los resultados emergentes de tales fuentes son coherentes entre sí y apuntan en la misma dirección. Para elaborar una explicación se acude a la identificación de factores de estructuración del campo de fuerzas intervinientes en las transformaciones lingüísticas y se postulan procesos a través de los cuales esas fuerzas van dirigiendo el cambio en el sentido indicado.

\begin{abstract}
How has the Aymara language changed among the indigenous people in Arica and Tarapaca for nearly a century and a half of republican life from its dominant language status to a subordinate condition in fast retreat before the Spanish language? In this article, the current status of this language is diagnosed and a historical explanation about the principal changes is developed. Diagnose and explanation are based on a socio-linguistic survey and on historical and ethnographic information. Results coming from such sources are coherent each other and both aim at the same direction. The explanation is developed by means of structuration factors of participating forces in the linguistic changes; besides, processes governing changes are postulated.
\end{abstract}

\section{Introducción}

El viajero y explorador Bollaert, refiriéndose a las poblaciones autóctonas que conoció hacia 1826 y que volvió a visitar, posiblemente, algunas décadas después durante un segundo viaje a Perú, señala enfáticamente que "los indígenas de la Provincia de Tarapacá son aymara y hablan esta lengua" (1975 [1860]: 474). Los aymara que conoció durante sus viajes y recorridos fueron principalmente aquellos de la precordillera andina tarapaqueña (Mamiña, Camiña, Tarapacá o Chiapa), aun cuando también visitó el altiplano de Isluga. ${ }^{2}$ La población de este grupo étnico en el área de Tarapacá ascendería, según sus cálculos, a unas 6000 personas, mientras que la población total, hacia 1860, llegaría a unos 18000 individuos (1975 [1860]: 465, 477). Por lo tanto, durante la primera mitad del siglo pasado, en Tarapacá ${ }^{3} \operatorname{los}$ aymara representaban alrededor de un tercio de sus habitantes y, aparentemente, todos hablaban el aymara, siendo con toda probabilidad un buen número de ellos monolingües.

En la actualidad la situación es por completo diferente. La aymara representa en la I Región un pequeño porcentaje de la población, aunque todavía prevalecen ampliamente en las zonas rurales, con un $75.5 \%$; en el conjunto de la región los aymara apenas alcanzan a $12.2 \%$ aproximado de la población. De ellos, según nuestros estudios, mantendrían algún grado de conocimiento del aymara sólo $44.5 \%$ de los individuos. Los porcentajes relativos más altos se encuentran en las zonas rurales y los más bajos en las ciudades costeras. Como es obvio suponer, en contextos de mayor presión hacia el recambio lingüístico, tal cual ocurre en los centros urbanos regionales, una proporción relativamente menor de aymara mantiene su lengua ${ }^{4}$. Además, ya son prácticamente todos hablantes del castellano: son monolingües castellanos $63.5 \%$, mientras que los bilingües aymara-castellano $\mathrm{y}$ unos pocos monolingües aymara llenan el restante

1 Instituto de Investigaciones Arqueológicas y Museo, Universidad Católica del Norte, San Pedro de Atacama, CHILE.

2 La extensión del conocimiento y uso de la lengua aymara por aquel entonces en la zona andina de los territorios de Tarapacá puede colegirse de la anotación realizada por Bollaert respecto a Mamiña: "es un gran pueblo indígena donde sólo se habla el aymara" (1975 [1860]: 476). Este poblado y los de sus inmediaciones son hoy día monolingües del castellano y, con excepciones, sus pobladores, descendientes de los indígenas de principios del siglo pasado, reivindican un pasado no aymara o, a lo más, mestizo.

3 Aproximadamente lo que hoy es la Provincia de Iquique.

4 Aun cuando, debido a la magnitud de las migraciones rural urbanas regionales, las cifras absolutas resultan bastante altas. En cualquier caso, más altas que en el altiplano o que en la precordillera. 
44.5\%. El mejor síntoma de los cambios acaecidos durante estos últimos 170 años lo representa el peso de los hablantes exclusivos de aymara a principios del siglo XIX ("donde sólo se habla el aymara") y a finales del siglo XX (donde los monolingües aymara son apenas unas decenas de ancianos aislados en la alta cordillera) (Cuadro 1) ¿Qué ha pasado en el intertanto? ¿Cómo se ha verificado tan importante proceso de cambio lingüístico? ¿Cuáles son sus causas principales? ¿Son éstas sólo, o principalmente, externas? La sociedad indígena regional ¿qué procesos de transformación ha vivido y respecto de qué dimensiones de su vida social pueden ser puestos en relación los cambios lingüísticos? ¿Cómo se relaciona todo esto con temas actuales tales como el de la etnicidad y el de la identidad étnica? En este trabajo queremos aportar información y elementos de juicio respecto a algunos de estos temas, abordando la cuestión del reemplazo lingüístico aymara en el área de la Provincia de Arica y Parinacota. Específicamente, trato de establecer por qué caminos y condiciones ha transitado el recambio lingüístico aymara durante el presente siglo. Para ello se utilizan los resultados de un estudio sociolingüístico que realicé en la zona hace algunos años. ${ }^{5}$

El material principal proviene de 212 entrevistas sostenidas con padres de familia o representantes adultos de otros tantos hogares, correspondientes a 22 localidades de la zona altiplánica y de la precordillera andina en las mencionadas provincias. A partir de ellas se cuenta con antecedentes y testimonios que permiten entender con relativa claridad cómo, durante los últimos 80 o 90 años, el avance del castellano se hace cada vez más masivo y profundo y cómo, paralelamente, el aymara se retrotrae en términos cuantitativos y se relega a espacios comunicativos intraétnicos cada vez más específicos.

\section{La distribución del bilingüismo}

La situación lingüística en la zona de estudio y, en general, en los diferentes sectores rurales y barriadas urbanas donde se concentra población aymara, se presenta extremadamente variada. Ella toma la forma de un continuo desde el monolingüismo aymara al

5 Este estudio se efectuó entre 1985 y 1986 en el marco del proyecto de investigación "Percepción de la escuela en la sociedad andina, características culturales (norte de Chile)" coordinado por Jorge Hidalgo a base de un convenio entre el Instituto de Antropología de la Universidad de Tarapacá en Arica y la Fundación Interamericana (IAF). monolingüismo castellano, pasando por una serie de situaciones intermedias: bilingüismo incipiente respecto al castellano, bilingüismo subordinado respecto al castellano, bilingüismo coordinado, bilingüismo subordinado respecto al aymara y bilingüismo incipiente respecto al aymara. Los extremos parecen estar bastante claros. A modo de referencia, señalamos que en el área estudiada los monolingües aymara representaban $3.4 \%$ de la población entrevistada, en tanto que los monolingües del castellano llegaban a $22.1 \%$. En cuanto al bilingüismo subordinado, éste se presenta también con un balance claramente favorable al castellano. El bilingüismo subordinado respecto al aymara (mayor desenvoltura con el castellano que con el aymara) cubriría $44.1 \%$ de los casos en la zona de estudio; en tanto que la condición inversa, de bilingüe subordinado respecto al castellano (mayor desenvoltura con el aymara), se manifestaría en $31 \%$ de los casos. El bilingüismo coordinado, la situación ciertamente más deseable, mantendría $24.9 \%$ de los casos examinados. Las situaciones de bilingüismo incipiente del aymara y del castellano por cierto existen, pero son muy poco significativas en términos numéricos.

Según se colige del examen de la distribución del bilingüismo en distintos estratos de edad, desde por lo menos principios de siglo se viene produciendo un paulatino incremento en la competencia del castellano. Ello se expresa en un decidido aumento de los monolingües castellanos y de las formas de bilingüismo en las que se mantiene mayor competencia con el castellano que con el aymara. Al mismo tiempo, de manera paralela, se verifica un declive del aymara expresado en que hay cada vez menos monolingües, menos bilingües subordinados respecto al castellano y menos bilingües coordinados.

Pero, la anterior, aun cuando es una situación generalizada y generalizable a toda la zona aymara, está lejos de ser homogénea internamente. Similares efectos de un largo proceso de recambio lingüístico se hacen sentir por doquier entre los aymara, pero con un nítido desfase temporal según se considere la región precordillerana o el altiplano. En esta última zona, por ejemplo, recién hace un par de décadas se hace evidente la formación de un grupo de individuos monolingües del castellano, específicamente en el estrato más joven de la población.

Cómo fue configurándose el bilingüismo, entendido como etapa más o menos prolongada de una 
dinámica de recambio lingüístico, es la materia que abordo en los siguientes acápites.

\section{La adquisición de una segunda lengua: Situaciones y contextos}

\section{Adquisición simultánea del castellano} y el aymara

En el conjunto de la zona estudiada el aprendizaje de ambas lenguas al unísono se presentó para $12.5 \%$ de los casos considerados en el estudio. En el altiplano este porcentaje crece para llegar a $17.9 \%$, en tanto que en la precordillera decrece, dando cuenta de $6.4 \%$ de los individuos. En la primera de estas subzonas tiene un crecimiento sostenido como situación de aprendizaje de ambas lenguas (en el estrato de los 4-14 años llega a 30.6\% de los casos), mientras que en la zona agrícola manifiesta un comportamiento mucho más errático. El aprendizaje de ambas lenguas simultáneamente, tal parece, representa una situación de transición o situación bisagra dentro de un proceso de cambios más amplios. En el altiplano expresaría, todavía, la capacidad del aymara de reproducirse con cierta vitalidad debido a la masividad de quienes lo conocen. En la precordillera, es más bien un símbolo de la vitalidad que ostentó en el pasado y una contingencia. Símbolo en la medida que para la población originaria el aprendizaje simultáneo de ambas lenguas dejó de ser una realidad hace muchos años; contingencia porque los casos más recientes corresponden a migrantes provenientes de zonas aymarófonas más altas.

Ambientes familiares y comunitarios aymarófonos que facilitan el aprendizaje del aymara se encuentran hoy, preferentemente, en la zona altiplánica. Allí, además del empleo corriente o mayoritario del aymara en el hogar, se emplea también de manera regular en la interacción de los individuos y grupos de cada comunidad. En la región agrícola más baja encontraremos algunos hogares que emplean regularmente el aymara en su comunicación cotidiana interna, pero sólo de manera excepcional este uso se prolonga a los contextos comunicativos interfamiliares y comunitarios. En el altiplano, entonces, muchos niños durante sus primeros años de vida han aprendido tanto el aymara como el castellano aprovechando que entre sus padres, hermanos, familiares y vecinos se comunican con una y otra lengua. Posiblemente, el aprendizaje del aymara provendrá en lo principal de las madres y de los ancianos, en tanto que el castellano, de los hermanos, los adultos jóvenes y diversos miembros de la comunidad. Un reforzamiento decisivo de este último aprendizaje lo tendrán en la escuela.

Resulta claro que los ritmos, los matices y las variaciones de esta situación general pueden ser muchos. Podemos pensar, por ejemplo, que la presencia de abuelos en el hogar, el tener una madre bilingüe subordinada respecto al castellano o hermanos que suelen emplear el aymara, estimulará a que un niño adquiera inicialmente tanto, o incluso más, aymara que castellano. La ausencia de abuelos, el tener padres jóvenes que emplean de preferencia el castellano para comunicarse determinará, al contrario, un balance distinto de aprendizaje de ambas lenguas. Una comunidad en donde el aymara es de uso cotidiano, a pesar de que los miembros del hogar prefieran hablar el castellano, permitirá, todavía, que con el curso de los años un niño también aprenda aymara. O sea, allí donde, como en el altiplano, existen ambientes familiares y comunitarios aymarófonos se darán situaciones de aprendizaje de ambas lenguas en que el aymara permanece en un estatus no subordinado o, al menos, no con el carácter de lengua aprendida de manera incipiente.

Allí donde, por el contrario, encontramos ambientes lingüísticos en los que prevalece el castellano y en los que hay una débil vigencia de la lengua indígena, ${ }^{6}$ cuando se aprende, el aymara, tenderá a definirse como lengua subordinada o incipiente respecto a este último.

Visto en términos procesuales, apreciamos una inversión de las condiciones que hacían posible estas situaciones de aprendizaje simultáneo. Los resultados de las entrevistas tienden a mostrar que durante las primeras décadas de este siglo en la precordillera y hasta hace unos 30-40 años en el altiplano, el aprendizaje simultáneo de ambas lenguas, cuando se daba, tenía al aymara como lengua fuerte. Es decir, tendían a generarse situaciones de bilingüismo en los que el castellano estaba en situación de inferioridad respecto al aymara. Por cierto, ello estaba en relación con un uso de la lengua vernácula, tanto en los espacios domésticos como en contextos sociales más amplios, mucho más extendido que en la actualidad. Posiblemente,

\footnotetext{
6 Por ejemplo, lo que sucede ya en algunas localidades del altiplano y en prácticamente toda la zona agrícola, a excepción de grupos familiares particulares, normalmente de migrantes provenientes de Bolivia o del altiplano aledaño.
} 
desde muy antiguo se aspirara a conocer y dominar cabalmente el castellano, por su utilidad para ampliar los horizontes comunicativos y las posibilidades económicas más allá de los espacios intraétnicos, pero los progenitores y miembros de la mayoría de los hogares no tenían conocimientos o no empleaban esta lengua con la frecuencia suficiente como para favorecer un aprendizaje temprano del castellano, contemporáneo con el aymara. Con posterioridad a los momentos indicados, en uno y otro sitio el aprendizaje del aymara al unísono con el aprendizaje del castellano manifiesta condiciones más favorables para esta última lengua. Es decir, cuando el castellano ocupa la posición de lengua general y de prestigio, entonces, aunque el aprendizaje simultáneo de ambas lenguas se dé de manera frecuente, esta alta recurrencia tenderá a estar circunscrita a un cierto período, de transición, y de todas maneras tenderán a conformarse bilingües en los que el aymara se encuentra en posiciones de inferioridad y subordinación.

Hoy día la situación está resuelta en favor del castellano en la precordillera. Es decir, en términos generales no se está aprendiendo aymara. Las tendencias apuntan en el mismo sentido para el caso del altiplano. Más de $80 \%$ está aprendiendo castellano de manera exclusiva o simultáneamente con el aymara. Asimismo, no más de $48 \%$ de los niños aprende aymara, sólo o simultáneamente con el castellano.

En este último espacio, el aprendizaje más o menos simultáneo de ambas lenguas se está dando, a pesar de los esfuerzos de los progenitores y familia para que los hijos aprendan solamente el castellano. Con ello buscan evitar una serie de problemas en la escuela y en las relaciones con aymara en un mundo indígena que se torna crecientemente integrado. Pero, por otra parte, por vía de los hechos, el aymara se mantiene como un vehículo lingüístico usual en la familia y/o en la comunidad. De allí que, a contrapelo de los planes y deseos de los adultos, numerosos niños llegan a aprenderlo y, eventualmente, a emplearlo ya a temprana edad. En la actualidad, entonces, el aprendizaje simultáneo de ambas lenguas es a pesar de los deseos e intereses de los aymara que privilegian el castellano. Por tanto, mientras el aymara conserva un grado importante de vigencia, mantendrá la fuerza suficiente como para ser aprendido masivamente junto con el castellano, aun a pesar de la aspiración de muchos por ser monolingüe castellano.
Esta fortaleza es la que se ha ido debilitando. De allí que el aprendizaje del aymara, simultáneo o no con el castellano, dejará de producirse cuando disminuya aún más la posición del aymara como medio de comunicación corriente entre los aymara. Esta parece ser una cuestión no tan lejana bajo las actuales circunstancias.

El propósito consciente de los propios aymara por eliminar su lengua o, cuando menos, de dejarla en un subsidiario segundo plano, junto con las conductas concretas de represión lingüística que acompañan tales aspiraciones, en cuanto se trata de una actitud generalizada, inaugura una fase más contemporánea del proceso de reemplazo lingüístico acelerado que se vive en los Andes chilenos. Es una etapa en la que la mayoría de los factores que incidían en la reproducción de la lengua han desaparecido o perdido importancia y en la que los factores subjetivos de rechazo por sus propios usuarios empiezan a jugar un papel de primerísima importancia.

\section{Adquisición diferida del castellano}

El castellano es, en la zona andina estudiada, una lengua que, sobre todo en la actualidad, se aprende desde la niñez. En la muestra considerada en la encuesta se había aprendido como primera lengua en $50.2 \%$ de los casos o al unísono que el aymara en $12.5 \%$ de ellos. Estos porcentajes se acrecientan hasta completar casi $90 \%$ durante la última década y media.

La adquisición diferida del castellano presupone un estado de monolingües aymara temporales. Actualmente, la aparición de ellos es cada vez más improbable. Según puede desprenderse de algunos casos recientes conocidos, se trata de niños criados con padres, abuelos u otros parientes monolingües, normalmente con residencia distante de cualquier escuela y sin medios económicos para solventar estudios en otro sitio, sin parientes que los acojan o ayuden en el lugar donde podrían escolarizarse o sin interés de los adultos a su cargo para que se escolaricen. ${ }^{7}$ Es decir, aunque una cierta proporción

\footnotetext{
En el pasado es posible que una mayor proporción de estos individuos fueron mujeres. Esto ha ido cambiando en la actualidad. Aunque presentan como promedio uno o dos años menos de escuela y la proporción de analfabetas es mayor que los hombres, hoy hay una similar preocupación para que aprendan castellano y por enviarlas a la escuela.
} 
de aymara sigue aprendiendo su lengua, en prácticamente todos los casos aprenderán el castellano antes o durante los momentos en que se adquiere el aymara. ${ }^{8}$

Pero, esta situación global para el área de estudio se especifica de manera particular al interior de los pisos ecológicos que hemos venido estudiando. En la zona agrícola alta, $84 \%$ de los individuos considerados tenía la lengua castellana como primera lengua o la había aprendido en simultaneidad con el aymara. El 16\% restante había tenido como primera lengua el aymara. Las situaciones de aprendizaje del castellano con posterioridad al aymara, que a continuación se describen, tienen que ver, por lo tanto, con esta minoría. Ella está compuesta por un remanente histórico de aymara que adquirieron el aymara como primera lengua en la precordillera, durante momentos tan lejanos como las tres primeras décadas de este siglo (alrededor de 7\% del total) y por migrantes de pisos ecológicos superiores asentados definitivamente en la zona agrícola alta (alrededor de 9\% del total). ${ }^{9}$

Es decir, desde principios de este siglo por lo menos, en esta zona la mayoría de los individuos aprende el castellano cuando niño, ya sea como primera lengua o en simultaneidad con el aymara. Durante todo este siglo y, posiblemente desde antes en algunos sitios, no es una lengua secundaria para segmentos importantes de esta población. Avala lo anterior el hecho de que los individuos implicados en situaciones de aprendizaje tardío del castellano, en momentos en que ya son adolescentes o adultos, llegan apenas a $7.6 \%$ del total. Por lo demás, hay un aporte considerable de personas nacidas en pisos superiores y posteriormente radicados en la zona agrícola. Desde hace algunas décadas no hay casos de personas que realizan un aprendizaje del castellano después de la temprana niñez.

8 Los monolingües iniciales del aymara y la adquisición diferida del castellano es hoy casi un anacronismo. Resulta tan improbable, por la notable castellanización que llevan asociados los procesos de integración económica, cultural y lingüística a la sociedad nacional que viven los aymara.

9 Bajo el supuesto que entre los residentes en la precordillera nacidos en el altiplano y que aprendieron el castellano como segunda lengua se mantienen los porcentajes detectados para el altiplano en cuanto a un temprano aprendizaje del aymara.
Los contextos particulares en que se producía este aprendizaje ulterior del castellano son múltiples. En su exposición las distinguiremos según si se trata de población originaria o forastera. Se detectaron algunos casos de adultos mayores y ancianos que, siendo oriundos de la zona precordillerana, tuvieron por primera lengua el aymara, aprendiendo con posterioridad el castellano. Efectuaron este aprendizaje con miembros de sus familias, en la comunidad y en la escuela. Algunos ancianos entrevistados aprendieron castellano fuera de sus espacios de origen en la ciudad de Arica, en los valles agrícolas bajos o en faenas mineras y viales cuando eran adolescentes o adultos jóvenes. En todos esos sitios un requisito indispensable para poder comunicarse, era el castellano. De este modo podían quedar, en cuanto peón, bracero, "carrilano", obrero o empleada doméstica, menos expuestos a tratos y relaciones laborales desventajosas.

Sobre los migrantes de los pisos superiores recae la responsabilidad de haber dilatado la persistencia de la lengua aymara en la precordillera. Su presencia hizo posible un proceso de cambio lingüístico más gradual. Por poco significativo que sea este segmento en términos numéricos, es oportuno detenerse más en ellos por el papel destacado que han jugado en relación a su lengua.

Las migraciones más antiguas de hace 50-60 años, serían básicamente de monolingües aymara y bilingües subordinados del castellano. Ellos encontraban una comunidad que se comunicaba preferentemente en castellano. Este se empleaba, además del aymara, en el trabajo agrícola, en las relaciones sociales comunitarias y al interior de las familias en su comunicación cotidiana. Otro tanto ocurría en las relaciones entre niños y adolescentes. El castellano era la lengua en la que se impartía enseñanza en la escuela. En suma, había diversos contextos sociales, formales e informales, comunitarios, familiares o de relaciones interpersonales en los que los migrantes tenían abundantes oportunidades de trabar contacto con el castellano. Eran oportunidades de familiarizarse y empezar a comunicarse en esa lengua o de ampliar y mejorar su desempeño. Las presiones eran múltiples. Estaba la necesidad de manejarse en lo más básico con el castellano, para resolver múltiples asuntos en un ambiente donde éste era un medio de comunicación extendido, pero estaban además las presiones 
provenientes de la posición de prestigio que ocupaba y ocupa en relación al aymara y las derivadas de la baja valoración de la lengua indígena (lengua de "indios", signo de falta de "civilización", sinónimo de "atraso", etc.).

Con posterioridad a la etapa recién comentada, la condición lingüística de los campesinos migrados desde el altiplano boliviano y chileno a los valles agrícolas cambia de monolingües (los más antiguos) o bilingües incipientes, que apenas se hacían entender en castellano, a bilingües coordinados y a los primeros monolingües del castellano. Esto último sería un fenómeno reciente que afecta a niños y jóvenes migrantes. Es una consecuencia clara de la intensidad de los cambios que también vienen produciéndose en la zona altoandina desde la que provienen esos migrantes.

Parte de los migrantes provenía de Bolivia. Hombres y mujeres llegaban y llegan hoy normalmente solos, ya jóvenes o adultos, en calidad de peones. Aprendían o aumentaban su competencia en el castellano con la población local de las aldeas agrícolas. Algunos niños que llegaron acompañando a sus progenitores o parientes, tuvieron ocasión de asistir a escuelas chilenas donde también aprendieron castellano. Como en el caso de los campesinos provenientes del piso altoandino chileno, el aprendizaje del castellano propiamente tal se dio en los casos de las migraciones de más antigua data. Posteriormente, se crearon las escuelas bolivianas altiplánicas que empezaron también, como en Chile, a enseñar el castellano. De aquí que cada vez más los migrantes del país vecino estén llegando como bilingües. Los cambios lingüísticos que les acaecen en Chile se dirigen más bien hacia un creciente abandono y desuso del aymara -dinámica que no ocurre o se da de distinta manera en Bolivia- y a la adopción de la variante dialectal chilena del castellano.

Los hijos de los migrantes altoandinos, por su parte, llegan o llegaban a aprender algo de aymara como consecuencia de que en sus hogares y entre adultos, se hablaba y habla esa lengua con cierta frecuencia. Pero en esos hogares, al mismo tiempo, estaba y está muy presente la preocupación de enseñarles preferentemente castellano, inspirados en la convicción de que esta lengua les será más funcional tanto en la escuela como en la vida de adultos. La transmisión normal del aymara entre migrantes a la precordillera se ve entorpecida, entonces, en la primera generación de descendientes. Lo más probable es que esta disrupción vaya en aumento a medida que se examinan casos de migraciones más recientes y de migrantes jóvenes. ${ }^{10}$

En el altiplano es donde una mayor proporción de personas ha tenido al castellano como segunda lengua y la ha adquirido tardíamente. Todavía 26\% de su población la adquirió de adolescente a adulto. Los cambios, sin duda, están produciéndose y ellos se precipitan a ritmos vertiginosos, pero el balance global resultante es todavía el de una zona donde el aymara retiene vigencia e impronta visiblemente su realidad lingüística. En efecto, hasta hace unos 40 años, en el altiplano, la mayoría de las personas que aprendía castellano lo hacía después del aymara (alrededor de $90 \%$ de los casos) y lo hacía mayoritariamente como adolescentes o adultos (69.8\% de los casos). Posteriormente, la proporción de individuos que obtienen el aymara antes que el castellano baja rápidamente (hace una década o década y media llegaba ya a $17.2 \%$ ). Es decir, no menos de $83 \%$ de los niños está aprendiendo muy temprano el castellano, en su mayoría en condición de primera lengua. Lo más probable es que tales porcentajes se encuentren ya en $100 \%$ de los casos, a juzgar por el hecho de que en los estratos de edad 5-14 y 15-24 años, correspondientes a grupos de población actuales de entre 12 y 32 años prácticamente no estén apareciendo casos de aprendizaje del castellano en edad adolescente o adulta.

Las circunstancias en que ocurría este aprendizaje ulterior del castellano fueron variadas. Resumiremos algunas. Una relación directa habría entre las

10 Lo anterior empalma con cambios más generales. A diferencia de los originarios, este grupo de población precordillerana ha aportado nuevos aymara hablantes en momentos en que la lengua dejaba de usarse. No obstante, desde hace por lo menos 25 a 30 años; empieza a disminuir gradualmente el porcentaje de migrantes que habla con desenvoltura el aymara. Probablemente hay ya personas que llegan del piso ecológico superior y que no hablan fluidamente esa lengua. Se produce, asimismo, un redireccionamiento de las migraciones altiplánicas, que crecientemente tienen como blanco migratorio sectores agrícolas más dinámicos o directamente las ciudades portuarias de la región. Por tanto, el fenómeno de realimentación lingüística en la precordillera está llegando a su fin. Con ello, se está incidiendo en una aceleración mayor del abandono y desuso del aymara, así como en una disminución del número total de hablantes. El bilingüismo en la precordillera toma la recta final que conduce al término del aymara, pues desaparece uno de sus sostenes más importantes durante este siglo. Parece significativo a este respecto la ausencia de niños y jóvenes que en esta zona tengan el aymara como lengua materna y la disminución de niños bilingües a un mínimo en el estrato de edad 5-10 años. 
características del ambiente lingüístico imperante y la extensión, profundidad u ocasionalidad del aprendizaje del castellano. Es decir, mientras más fuertemente aymarófono fuera este ambiente, con mayor probabilidad se llegaría a tener acceso a un castellano, usualmente limitado y superficial, traspuesto una cantidad de años de la vida de los individuos. Un ambiente de este tipo al parecer existió en el altiplano hasta hace unos 50 años, a juzgar por las proporciones que para los nacidos en esos años entregan las entrevistas en cuanto a conocimiento del aymara, desempeño con el castellano, primera lengua y momento de aprendizaje del castellano. En todos estos indicadores se presentan proporciones que indicarían, todavía para ese entonces, una situación de conocimiento y uso masivos del aymara y un conocimiento y empleo limitado del castellano. En esta medida, la adquisición del castellano en el altiplano se restringía a familias que, excepcionalmente, hacían uso del castellano o, lo que sería más frecuente, a la obtención de algún vocabulario y de los primeros rudimentos de la lengua.

Hasta ese entonces la lengua castellana era aprendida por una baja proporción de sujetos, de manera superficial y, las más de las veces, en otros espacios étnicos o en contextos extraétnicos. La zona precordillerana es el espacio intraétnico más importante para este temprano acceso al castellano. Cuando los pastores de altura bajaban periódicamente a efectuar intercambio complementario y trabajos estacionales entraban en contacto con castellano hablantes en las labores de campo, en el trato con comerciantes, en las relaciones con vecinos, en los actos y reuniones sociales y festivas. No era extraño que hijos de estos pastores quedaran a cargo de familias pudientes, bajo el compromiso de alimentarlos, vestirlos, enseñarles castellano y hacerles asistir a la escuela si la había, a cambio de sus servicios como niños-peón. Excepcionalmente, campesinos de la Puna tenían algunos recursos como para enviar por temporadas o durante algunos años a sus hijos a escuelas existentes en los valles, sin necesidad de pasar por la servidumbre. ${ }^{11}$ Como resultado de esto se generaban bilingües incipientes del castellano o, en los casos de una interacción más frecuente o sistemática con el castellano, bilingües subordinados y quizá algunos de los

11 Escuelas de primeras letras existieron en la zona precordillerana desde principios de siglo. Por ejemplo, en Putre, Belén, Socoroma, Tignamar y Codpa. primeros bilingües coordinados aymara-castellano del altiplano.

Los espacios extraétnicos en que se efectuaba contacto y aprendizaje del castellano eran las escuelas de instrucción pública de otras zonas rurales andinas (valles de Lluta y Azapa), a las que llegaban niños en condiciones parecidas a las descritas para la zona agrícola alta; las faenas en la red ferroviaria Arica-La Paz, en las que solía asalariarse una cierta cantidad de mano de obra aymara; los campamentos mineros como los de Tacora y Choquelimpe, donde la población aymara aledaña convergía frecuentemente para comercializar carbón vegetal, llareta, carne y otros productos menores e, incluso, para emplearse temporalmente; los valles agrícolas más bajos, a los que se accedía con fines de intercambio complementario, comercialización de productos y asalariamiento temporal; las ciudades de Arica y Tacna, a las que se llegaba para efectuar tramitaciones o a trabajar por temporadas. Cabría destacar todavía, la fuerte presión que recibían los aymara que llegaban a los regimientos de la región o del sur del país a servir su conscripción militar para que aprendieran a hablar castellano, aprendieron lectoescritura, adhirieron conceptos, símbolos y valores patrios, etc. ${ }^{12}$ Otro tanto se venía efectuando, aunque de un modo menos sistemático y electivo, en las dotaciones policiales instaladas, primero, en la región precordillerana y, más tarde, en el altiplano. ${ }^{13}$ Muchos de estos factores, que aquí

12 Un aspecto del denominado proceso de "chilenización" de Tarapacá y Arica es el adoctrinamiento que la mayoría de los jóvenes aymara de la zona interior fueron recibiendo desde principios de siglo en regimientos instalados en Arica o Iquique o bien en el sur del país. Ancianos aymara recuerdan con nitidez, por experiencia propia o por lo ocurrido a padres y tíos, la fuerza con que se buscó doblegar sus anteriores lealtades nacionales (peruanas) y, de paso, sus lealtades étnicas (estas últimas bajo la óptica de "civilización”).

13 Fuera de la zona de estudio, las comunidades de la precordillera de la Provincia de Iquique mantuvieron desde el siglo pasado intensas vinculaciones con la pampa salitrera, satisfaciendo una importante demanda de productos campesinos e insertándose en el mercado de fuerza de trabajo asalariado minero. Tan profundas llegaron a ser estas relaciones, que se las encuentra normalmente como telón de fondo a las transformaciones agrarias, sociopolíticas y culturales que ha vivido la zona. Aunque entre pares aymara era siempre posible comunicarse en la lengua vernácula, la lengua de comunicación general era el castellano, por lo que a los monolingües aymara o bilingües subordinados respecto al castellano, o a los hablantes del quechua, les era ventajoso aprender o mejorar su desempeño con él. Este impacto rápidamente se extendía a otros segmentos de las comunidades. 
presentamos desagregados por el análisis, actúan en la vida cotidiana de los individuos aymara de manera simultánea o en la forma de conjuntos encadenados de factores. Algunos ejemplos pueden ilustrar adecuadamente esta situación: un joven recién casado que ha terminado algún tiempo atrás su ciclo de recluta, período en el cual se le reparó su fonética, relativa pobreza de vocabulario castellano y la variante dialectal del castellano que empleaba, impactado por la carga peyorativa que estas experiencias hacían recaer sobre su persona, seguramente exigirá a su mujer alcanzar niveles de desempeño semejantes a los que él adquirió, con el objeto de ponerla a resguardo de la mofa y del desprecio cuando terceros, particularmente en medios urbanos, reparan su condición étnica a partir de su desenvolvimiento lingüístico. Otro ejemplo: lo más común entre aquellos que en el altiplano tienen por lengua natal el aymara, es que aprendan, en mayor o menor grado, el castellano en la familia, aprendizaje que, a medida que pasan los años se complementa en la comunicación con otros niños y miembros de la comunidad. Cuando tenga edad para ello, en la escuela, se verá forzado a complementar y reforzar lo ya adquirido, hasta desenvolverse satisfactoriamente con las materias que se le enseñan. Otro caso puede ser el de adultos mayores y ancianos que en el pasado aprendieron los primeros rudimentos del castellano en sus viajes de intercambio y trabajo a otras zonas campesinas, conocimiento que ampliaron cuando sus hijos escolarizados empezaron a emplear el castellano en la comunicación diaria entre ellos y con los miembros del hogar que supieran algo de castellano. Sus conocimientos, simultáneamente, se fueron afianzando en la comunicación con otros campesinos sometidos a similares influencias, que en algunas ocasiones preferían el castellano para comunicarse entre ellos. Sin duda, hay una multiplicidad de procesos individuales particulares en la adquisición del castellano, donde se combinan y actúan múltiples factores interconectados.

Las oportunidades para el aprendizaje del castellano estaban principalmente centradas, entonces, en los adultos varones que manejaban las relaciones con el exterior. En segundo orden de importancia estaban los adolescentes, también varones, que iniciaban con sus mayores sus ciclos de aprendizaje de la vida laboral, económica y social de pastores en los pisos y enclaves extraétnicos. Por último, como un segmento más reducido, están los niños que ocasionalmente eran dejados con terceros como peones o pensionados. Las mujeres, por su parte, solían estar aparte de estas posibilidades de aprendizaje. Por lo tanto, durante la primera mitad de este siglo fue paulatinamente aumentando el número de aymara del altiplano con capacidad para comunicarse con la lengua castellana, en la mayoría de los casos de una manera insuficiente dada su condición de bilingüe incipiente o subordinada. Un mayor número de hablantes y grados mayores de conocimiento habrían permitido al final del período que comentamos, el inicio de una comunicación "normal" en castellano, preferentemente entre hombres aymara.

Aproximadamente hasta 1950 la penetración del castellano parece haber sido selectiva y especializada. Selectiva, en la medida que impactaba de preferencia a ciertos estratos etarios y a los hombres. Las mujeres estaban ligadas a las labores hogareñas y al pastoreo en lejanas estancias y majadas de la Puna. Especializada, en la medida que era un instrumento de comunicación todavía no indispensable en las relaciones entre aymara del altiplano. Se empleaba para la comunicación con un cierto segmento de población de los valles que se comunicaba en castellano y, sobre todo, para posibilitar relaciones de diversa índole con no aymara. El castellano era, en esta medida, una lengua secundaria e instrumental manejada por un grupo o segmento particular de los miembros de la comunidad.

Al parecer, tampoco estaba presente en el altiplano, ahora en una dimensión subjetiva, la polivalencia de sentido que hoy ostenta el castellano. Desde la lectura que posibilitan ciertas actitudes aymara de ese entonces, una de las fuerzas operantes sería una tendencia centrípeta de repliegue y defensa. Visto desde esas actitudes y haciendo abstracción de otras tendencias contradictorias, se trataría en términos culturales de una zona de refugio, de una región aislada y relativamente conservadora que se desenvolvía principalmente en circuitos sociales y económicos campesino e intraétnicos. Sería una fracción de la sociedad aymara que no aspiraba, por importancia o por opción, a procesos de integración mayores que los que ya vivían, eventualidad en la cual era vital disponer del castellano, una herramienta básica para la comunicación recíproca. ${ }^{14}$

14 A contrapelo de ello, se hace hoy impensable para un aymara no saber castellano. No saberlo significa aislamiento social, ausencia o disminución de oportunidades de vida, trabajo y progreso; ignorancia, retardo o atraso en cuanto al conocimiento del mundo actual y de lo que ocurre más allá de las 
La segunda mitad del siglo trae fuertes cambios en la suerte del castellano en el altiplano. Deja de ser una lengua de acceso selectivo y con funciones especializadas, para transformarse en una lengua que se aprende de manera generalizada y que presta funciones comunicativas múltiples. Llegará rápidamente a ser de uso general, la principal en el altiplano. Este estatus lo había alcanzado en la precordillera durante el medio siglo anterior.

El castellano empieza a ser obtenido por una cada vez mayor proporción de individuos y a edades cada vez más tempranas. Esto es así al grado que un $100 \%$ de los individuos ubicados entre cinco y 14 años considerados en el estudio habían aprendido castellano durante ese lapso, antes que el aymara $(52.2 \%)$, junto con él $(30.6 \%)$ o inmediatamente después de él (17.2\%). ¿Qué ha sucedido? Una discusión se entregará en el acápite siguiente. Nos limitamos aquí a señalar ciertos elementos y contextos en los que se producen estos cambios.

Por cierto, durante los años siguientes a 1950, los factores y contextos de aprendizaje del castellano que se examinaron para la primera mitad del siglo siguieron estando vigentes. A través de ellos se siguió ampliando la cobertura de los castellano hablantes y estos factores, probablemente, también contribuyeron al mejoramiento de la competencia en el uso de la lengua para muchos aymara. Sin embargo, aparecen nuevos agentes y situaciones que operan como multiplicadores y catalizadores de la expansión del castellano. El más importante es la escuela pública nacional, cuyos establecimientos empezarán a instalarse en el altiplano y a formar una red de escuelas, a las que obligatoriamente asistirá la gran mayoría de los niños aymara, hombres y mujeres, monolingües o bilingües, con o sin el consentimiento de los padres, para recibir instrucción en lengua castellana. Por aquel entonces, poseer escuela en el mismo altiplano era una sentida demanda campesina, particularmente de los adultos jóvenes, más imbuidos y sensibilizados alrededor de ideas y estereotipos de progreso, desarrollo, integración, asimilación y nacionalidad.

fronteras de las comunidades de la puna; significa exponerse al estigma y al prejuicio étnico y racial sin contar con medios mínimos para defenderse; lo expone a la acusación gratuita de extranjero de poco valer ("boliviano"); significa ser "indio" necesitado de "civilizarse".
El acceso a educación formal para los hijos era representado y se representa hoy como una de las más poderosas palancas de cambio y mejoría de su condición sociocultural y económica.

Presiones y estímulos para la adquisición del castellano por quienes no lo poseían y el mejoramiento de las habilidades de los bilingües se han hecho sentir desde hace mucho, a través de la acción de profesores, policías, militares, empleados municipales, entre otros. Un papel destacado en la castellanización de la zona ha tenido la escuela y otras instituciones del Estado que se instalan en el Area Andina así como diversos agentes del mercado (comerciantes de productos de consumo básico, compradores de bienes campesinos, transportistas, etc.), todo ello unido a condiciones de comunicación, acceso y transporte que mejorarán progresivamente y facilitarán la fluidez de las relaciones sociales y económicas entre aymara y no aymara. Rápidamente, el castellano dejará de ser el patrimonio de los hombres y se asentará en lo más íntimo de la vida doméstica y femenina. Lograda su generalización dejará de ser una lengua para las relaciones interétnicas y rápidamente, en un par de décadas, llegará a ser también una lengua para las relaciones intraétnicas.

\section{Adquisición diferida del aymara}

El aprendizaje del aymara posterior al castellano aparece representado por dos situaciones sociolingüísticas límites y en dos extremos del período de tiempo que estamos considerando (alrededor de un siglo). La primera corresponde a finales del siglo XIX e inicios del XX, como momento en que se expresaba posiblemente con mayor intensidad. En aquel entonces la mayoría de la población de la zona precordillerana hablaba el aymara y el bilingüismo, que lo había, es posible que en la mayoría de los casos fuera subordinado respecto al castellano. Es decir, el aymara era aún mayoritariamente la primera lengua y el uso del castellano no estaba mayormente extendido. Hay un grupo de esta población, sin embargo, que había empujado el proceso más allá del resto. Es un segmento de la población andina de esa zona que en su medio familiar tenía al castellano como lengua de uso más frecuente, como lengua con la que se aprendía a hablar, pero que por diversas circunstancias se veía obligado a aprender el aymara. 
Se trata del primer estrato de población que en los valles altos había reemplazado el aymara por el castellano y había ubicado su universo de relaciones sociales más prestigiosas e importantes al interior de su propio grupo y con no aymara de los valles bajos y la costa. O sea, se trata de aquéllos que habían iniciado un despegue de la condición indígena y de campesino menesteroso, amparados en la tenencia de suficientes tierras y animales y en la posesión de capital para actividades de arriería y comercio. Podía tratarse, asimismo, de miembros de este mismo grupo al que aludimos, pero cuya proveniencia no es el enmascaramiento de la condición de indígena en la misma comunidad logrado con el paso de las generaciones. Eran migrantes mestizos, aliados por parentesco e intereses con los restantes miembros de las élites de poder locales, provenientes de lugares localizados dentro del circuito de las relaciones económicas y sociales de este estrato campesino más pudiente y social y étnicamente diferenciado (valles bajos, enclaves mineros, Arica).

Las circunstancias que los llevaban a la adquisición ulterior del aymara eran de dos tipos. Una que pudiéramos calificar de aprendizaje por osmosis, por efecto de una comunidad que solía desenvolverse mayoritaria o frecuentemente en aymara. Los niños y adolescentes que tenían al castellano por primera lengua estaban en frecuente contacto con el aymara, al oírlo hablar por los mayores y al escucharlo en los grupos de pares. El aymara aparecía en las faenas colectivas, en las reuniones sociales, en los intercambios con los pastores de pisos altos, en la comunicación con los peones aymarófonos, en las fiestas religiosas, en los ritos andinos. Paulatina y progresivamente, entonces, se iba conociendo y entendiendo la lengua indígena hasta que se daba el paso de empezar a hablarla, aunque fuera rudimentariamente. En un primer momento, se trata por cierto, de bilingües incipientes o, a lo más, subordinados del aymara.

Este aprendizaje inicial era la base de una segunda situación de aprendizaje complementaria y ulterior. Ya no será un aprendizaje involuntario y circunstancial, sino uno motivado por necesidades prácticas. En efecto, la vida económica de los valles altos estaba íntimamente ligada con los pisos superiores altiplánicos, tanto de Chile como de Bolivia. Eran economías campesinas complementarias que articulaban a través del intercambio estacional de productos y el asalariamiento (generalmente por productos y servicios) de peones aymara en los valles. Los campesinos que hacían contraparte eran básicamente monolingües. En estas circunstancias, una condición de la complementariedad es la comunicación lingüística que no podía darse sino en lengua aymara. Individuos aymara de los valles que no hablaron el aymara como primera lengua, sino el castellano, se veían llevados muy pronto a aprender sus rudimentos para hacer posible un grado mínimo aceptable de comunicación con la comunidad y con el sistema regional de relaciones campesinas del que formaban parte. Una situación de este tipo ejemplifica bien una primera fase del cambio lingüístico: la del castellano como lengua secundaria funcional y la del aymara como lengua general de la etnia.

En el otro extremo del período que analizamos y principalmente en el altiplano, ya que en la precordillera en la mayoría de los casos no se está aprendiendo aymara, encontramos la situación de niños que aprenden primero castellano $(52.2 \%$ en el estrato de edad 5-14 años) y después aymara. En esta situación estaría alrededor de $40 \%$ de los casos del estrato. Es decir, hay una fracción importante de los niños y adolescentes aymara del altiplano que, aunque no tienen por primera lengua el aymara, en su mayoría llegan a adquirir un grado mayor o menor de conocimientos de su lengua y, posiblemente, muchos de ellos la llegarán a emplear de manera regular u ocasional. Esto se ha venido produciendo en el altiplano desde hace unos 35 años, pero se ha incrementado notoriamente durante las últimas dos décadas. Ocurre algo semejante a lo que en su momento se fue dando en la zona precordillerana hacia fines del siglo pasado y principios del actual: hay un cierto momento en que el castellano rompe las barreras que contenían su expansión y, pasando por una fase transicional de relativo equilibrio con el aymara, se hace prevaleciente en la comunicación linguística al interior de la etnia. Con anterioridad denominábamos a esta situación el paso de una lengua de conocimiento restringido y uso especializado a una de conocimiento generalizado y uso múltiple.

A partir de la generalización del conocimiento, del uso y de los contextos del castellano, empieza a ganar terreno como primera lengua y los tipos de bilingüismo se amplían y complejizan para incluir también la subordinación del aymara, que antes no 
existía sino en la forma de casos excepcionales. Si la lengua castellana es de uso diario entre adultos, entre niños, si esta es la lengua de la escuela, la lengua de la comunicación con los forasteros y los no aymara, si el prestigio y la necesidad de ella están sólidamente asentados en la conciencia y las preferencias aymara, entonces, no puede ser sino normal que el aymara todavía practicado (con las abuelas, los mayores y los ancianos, con los campesinos aymara de Perú y de Bolivia, en el rito, etc.) se aprenda junto con el castellano o después de éste, y no antes que el castellano como ocurría en el pasado inmediato. Este tipo de situaciones ejemplifica la tercera fase del proceso de cambio lingüístico que examinamos: la del castellano como lengua general de la etnia y la del aymara como lengua secundaria empleada para fines cada vez más especializados.

\section{Conclusiones}

El castellano es la lengua nacional y aquella que domina en todas las dimensiones cruciales de la vida pública: comercio, educación, administración, justicia, política, literatura, medios de comunicación de masas, la producción científica, etc. Muy por el contrario, las lenguas nativas en Chile son lenguas regionales y sus funciones específicas se limitan a los espacios intraétnicos. ${ }^{15}$ Desde este punto de

15 Chile es un país multilingüe. Junto con el castellano, la lengua oficial y dominante, mapuche, pascuences y aymara conservan lenguas propias distintivas. Cabría recordar también el italiano, yugoslavo, alemán, etc., practicado por migrantes de esas nacionalidades $\mathrm{y}$, a veces, sus descendientes. Aunque la situación de unas respecto a otras de estas lenguas puede ser equiparable en algunos aspectos sociolinguísticos, ya sea por su origen, procesos sociales que han vivido o estatus, la realidad de las lenguas nativas es por completo diferente. Las otras comparten en algún grado el estatus privilegiado del castellano. Gozan, en primer lugar, de un prestigio notoriamente más elevado y de la protección y hasta el fomento por parte del Estado. Somos un país, como todos en Latinoamérica, en que se ha valorado dotar de instrumentos de comunicación necesarios para relacionarse con el hemisferio norte y, dentro de éste, con el llamado primer mundo. Somos básicamente una cultura europocéntrica, que se nutre culturalmente de los países desarrollados. El inglés es obligatorio. Se propicia el aprendizaje de una segunda lengua extranjera, francés o alemán. Hay escuelas italianas, alemanas, inglesas, francesas. Se traen profesores para la enseñanza de esas lenguas. Quien no sabe inglés es cada día una persona "menos culta" y con menos oportunidades. vista, todas ellas se encuentran en una situación de diglosia, en el sentido clásico del término. ${ }^{16}$

Hablan el aymara exclusivamente los aymara y, salvo excepciones, en el norte de Chile a nadie no indígena parece importarle su suerte. Pocas personas, además de los inmediatos interesados, parecen estar informadas de su existencia. Cuando la identifican, suelen mantener apreciaciones erróneas y prejuiciosas ("dialecto", lengua de indios, de los "bolivianos", algo fuera de época, extemporáneo y superado por los tiempos, etc.). Los juicios que se emiten rebajando su condición de lengua, remitiéndola a personas de menor valía social ("indios") o extranjeros (bolivianos) y definiéndola como algo caduco y sin validez en cuanto instrumento de comunicación, habla de las actitudes que en la región se tienen con el aymara.

El aymara es en el contexto regional y nacional una lengua desconocida y despreciada. Quienes han advertido su existencia no le otorgan un sentido y función más allá de su uso por la propia etnia. Al interior de esta misma hay una fuerte tendencia en favor de su abandono. ${ }^{17} \mathrm{El}$ aymara y el castellano en la sociedad regional son lenguas que no se tocan, que se excluyen mutuamente, que no se interfieren, son mundos paralelos salvo en las vivencias de los propios aymara. Aun para ellos este paralelismo es una realidad. La relación entre ambas lenguas está lejos de ser la de un bilingüismo total que permita su intercambiabilidad en todo tipo de contextos. La situación se ubica más bien en el otro extremo, el de contextos de uso claramente diferenciados y cada vez más específicos en el caso del aymara.

Al estatus general de lengua secundaria y abandonada a la suerte de lo que puedan hacer en su favor un sector de los propios aymara, acompañan tendencias de transformación que amenazan hacerla

16 Situación entre lenguas y dialectos de una lengua cuyo estatus y funciones varían desde una modalidad alta o prestigiosa, oficial y empleada en la educación, la literatura, los medios de comunicación, las leyes y la ciencia, a otra baja o minusvalorada empleada en la vida familiar o cotidiana. Mientras la modalidad alta puede ser empleada en el hogar, es muy improbable o poco frecuente que ocurra a la inversa. En el caso que nos preocupa, la oposición es siempre entre lenguas amerindias y una lengua nacional de origen exterior (castellano, portugués).

17 Que hoy día coexiste, afortunadamente, con otra que pugna por su mantención y, en ciertos contextos, por su recuperación. 
desaparecer en el largo plazo. En efecto, la lengua aymara en el norte de Chile está envuelta en un profundo y prolongado proceso de cambios, cuyo derrotero general es el de las lenguas en estado de diglosia: terminan por desaparecer. Este fenómeno de reemplazo lingüístico se está expresando en fenómenos que examinamos en páginas anteriores.

Primero, en una disminución importante del conocimiento del aymara. Ello se da tanto en términos absolutos (aumento del número de individuos que terminan por conocer prácticamente nada de su lengua) como relativos (incremento de individuos cuyos conocimientos disminuyen en amplitud y profundidad). Como se destacó, esta disminución es notoriamente más acentuada en la zona precordillerana. Esta progresiva disminución de conocimientos sobre su lengua que evidencian los aymara se expresa, entre otros fenómenos, en una merma rápida de los monolingües aymara y de los bilingües subordinados respecto al castellano. Se ve reforzado, en cambio, el monolingüismo castellano y las situaciones de subordinación del aymara.

En segundo término, se verifica una aceleración o progresión geométrica del ritmo de cambio lingüístico. La evolución del bilingüismo y el desarrollo del monolingüismo castellano entre los aymara es decidor al respecto. Hay una verdadera explosión en el aprendizaje del castellano como primera lengua, de los monolingües castellanos y de los bilingües incipientes respecto al aymara. Esta es una cuestión especialmente visible en el altiplano, donde el predominio del aymara era indiscutible hasta hace algunas décadas. Por ello, aun cuando la distribución del uso de ambas lenguas pueda ser excluyente, un bilingüismo estable no parece por ahora posible ni perdurable en el tiempo para el caso aymara. El que existe no es un bilingüismo estacionario, sino transicional. No estamos hablando de dos lenguas cuya posición en el seno de la población aymara sea estática. En este contexto, el bilingüismo aymara-castellano es un paso para llegar al monolingüismo castellano, puesto que éste se da en el marco de un fenómeno de "castellanización". ${ }^{18}$

18 Por castellanización entendemos un proceso de reemplazo lingüístico que involucra al castellano y una segunda lengua, donde la primera ocupa una posición hegemónica y dominante y la segunda una subordinada. La primera se impone y reemplaza a la subordinada. En esta medida, se trata de una forma regional de diglosia donde interviene, por una parte,
Como tercera cuestión, la lengua aymara disminuye su funcionalidad. Visto a largo plazo, hay un proceso de cambios y redefinición de las funciones sociales de la lengua nativa. Según la evidencia disponible, su empleo es cada vez menos frecuente y la variedad de contextos sociales en que se la utiliza también está disminuyendo. A veces se usa como lengua del hogar, para la comunicación con los abuelos y a veces como código preferente respecto a campos semánticos particulares. De manera general, puede decirse que de lengua usada de manera generalizada en la comunicación entre los aymara pasa a ser una que todavía es patrimonio de la etnia, pero a cuyo interior satisface funciones comunicativas y simbólicas cada vez más particulares y especiales.

En cuarto lugar, la lengua aymara no sólo pierde funcionalidad y presencia en favor del castellano, sino que también valoración y prestigio por parte de sus usuarios históricos. Los antecedentes sobre el particular son abrumadores. Se ha asumido el bajo estatus implícito en la definición de dialecto con la que usualmente la población regional se refiere al aymara. Su posesión forma parte del estereotipo peyorativo de "indio" y éste es, por lo general, vivido como un estigma. No es casual, entonces, que se subordinen sus cualidades a las del castellano; que se disminuya la frecuencia de su uso; que cuando se la posee se actúe con ella de un modo reactivo para encubrirse o mimetizarse ante actitudes prejuiciosas de terceros; que se evite su uso fuera de los contextos estrictamente indígenas; que con frecuencia se reprima su uso entre los niños y jóvenes; que los padres estén vivamente interesados en que sus hijos aprendan castellano como primera lengua, dejando el aprendizaje del jaqi aru librado a las circunstancias de los ambientes lingüísticos en que los niños y adolescentes aymara se desenvuelven; que por lo común no se tenga una conducta activa para promover su aprendizaje, sino que más bien al contrario, etc.

En suma, el aymara se encuentra sumergido en una crisis de vigencia y reproducción que se agrava con el paso de los años. Esta difícil situación es general a todas las regiones con población aymara, según el conocimiento que disponemos de cada una de ellas

el castellano y, por otra, una lengua indígena o extranjera, pero minoritaria, cuyos hablantes se encuentran desprovistos de poder y capacidades para imponer un bilingüismo equilibrado. 
y de acuerdo a los resultados obtenidos por otros investigadores. ${ }^{19}$ Se trata, pues, de un problema central, que visto en términos de larga duración, constituye una fase de transformaciones culturales y sociales de gran envergadura que amenazan con la disolución de la lengua.

\begin{abstract}
¿Al interior de qué campo de fuerzas sociales se fueron produciendo estas transformaciones? Un primer tema es lo ocurrido durante el siglo XIX. Es decir, qué cambios lingüísticos y de qué naturaleza se fueron acumulando hasta las postrimerías del siglo pasado, momento a partir del cual hemos podido documentar la suerte del aymara y el castellano entre la población indígena regional. ${ }^{20}$ No contamos con información suficiente como para plantear juicios taxativos sobre el particular, pero es posible proponer algunas hipótesis de trabajo.
\end{abstract}

La sociedad aymara durante el siglo XIX se diferencia $\mathrm{y}$ divide en un segmento de campesinos ubicados en los valles agrícolas del pie del desierto y la precordillera andina y un segmento de campesinos más aislados en la alta cordillera, principalmente ganaderos. Los primeros protagonizan un proceso de integración social y cultural a la sociedad criolla radicada en ciertos enclaves agrícolas, la pampa salitrera y la costa. En un plano cultural, es el camino del mestizaje, del encubrimiento de la condición de indio, del aprendizaje del castellano, de la construcción de una memoria y orígenes no aymara, de la adopción de bienes y símbolos culturales prestigiosos provenientes de la sociedad de referencia. En lo sociopolítico, es el tiempo de la disolución legal de las comunidades históricas y los ayllu, de la descomposición de la "república de indios", del primado de la pequeña localidad sin subordinación o solidaridad con otras similares o respecto a un orden indígena superior. ${ }^{21}$ En lo económico es el tiempo de la formación en el agro andino de "hacendados", relacionado con la llegada de no indígenas o de

19 Por ejemplo, S. González (1990).

20 Principalmente a través del recurso a la memoria oral, de los resultados de las entrevistas realizadas durante el estudio que fundamentan este trabajo y de algunas fuentes secundarias.

21 Por cierto, no queremos insinuar que los factores componentes y las tendencias de transformación en el sentido de una integración a la restante sociedad regional hayan aparecido sólo en el siglo XIX. Por ejemplo, ciertas cuestiones como la descomposición de los cacicazgos regionales y la recomposición de formas de organización y poder alrededor de la comunidad aldeana son de finales del siglo XVIII. fenómenos de diferenciación interna y concentración de tierras y aguas; es el momento en que empieza a efectuarse un definitivo redireccionamiento de la economía indígena, particularmente de su estamento pudiente y de los mestizos insertos en las comunidades, hacia las producciones para un mercado de productos agrícolas y campesinos en formación en el desierto y la costa; es un período en el que cobra nuevos bríos la inserción del campesinado aymara en el mercado de fuerza de trabajo minero que se va consolidando en la zona, una parte del cual se desarraigará de la tierra y conformará, con grupos de individuos de otras proveniencias, el proletariado minero nortino.

El segundo segmento se mantendrá básicamente monolingüe hasta avanzado el primer cuarto de este siglo y en ciertas zonas, incluso, hasta más tarde. Su relación con la sociedad no indígena de los valles bajos, pampa y costa estará, por lo común, mediada por aymara de la zona agrícola donde, como se dijo, venían produciéndose fenómenos de diferenciación interna, redefinición de identidades e integración cultural y socioeconómica. Tal mediación es principalmente de tipo económico y sociopolítico. ${ }^{22}$ No tenemos evidencia de procesos de cambio en el altiplano que durante el siglo XIX fueran produciéndose con la intensidad y profundidad de la de los valles. Los pastores del altiplano, a resguardo por su relativo aislamiento, mantendrán en lo sustancial una cultura y forma de sociedad conformadas durante los siglos de coloniaje; sólo bastante más tarde, durante las décadas más recientes, se precipitarán las transformaciones que envuelven el mundo aymara de hoy.

Por tanto, durante el siglo XIX asistimos a una notoria diferenciación de la sociedad indígena

22 En el primer caso, a través de la formación de un estrato de rescatistas aymara y no aymara, asentados en ciertos valles, desde donde controlaban la actividad agropecuaria y abastecimiento de productos en zonas urbanas o enclaves mineros. En el segundo, muchas de las relaciones entre las comunidades altiplánicas y las autoridades y administración del Estado peruano parecen haber estado mediadas, precisamente, por el estamento de agricultores, rescatistas y comerciantes que se habría ido formando en las cabeceras de los principales valles agrícolas de la zona. Para unos, estos individuos estaban provistos de relaciones y ciertas influencias útiles para tener acceso a las autoridades y funcionarios, además de hacer posible la comunicación con ellos. Para otros, además de servir de intérpretes, tales personajes contaban con un conocimiento de la geografía y los problemas de la alta cordillera. 
regional. En términos lingüísticos esto significó una diferenciación paralela entre un altiplano monolingüe y una zona de valles crecientemente bilingüe y, más adelante, monolingüe castellana. ${ }^{23}$ Esta sería la explicación histórica a las notorias diferencias lingüísticas que se evidencian desde fines del siglo pasado y que se prolongan hasta hoy en una y otra zona. ${ }^{24}$

Un segundo tema es el del mercado. Es la cuestión de la inserción de las economías aymara en sistemas económicos regionales. Aunque desde tiempos coloniales no se trata de economías totalmente ajenas a los sistemas regionales, tampoco son, hasta aproximadamente la segunda mitad del presente siglo, economías integradas con fuerza hacia el exterior. Esta integración se ha dado principalmente en el mercado de fuerza de trabajo regional, y en el mercado de productos campesinos. Más recientemente, en el último siglo, ha cobrado relevancia el mercado de bienes y productos de consumo humano. Todo esto ha significado relaciones más o menos continuas, según los casos, con poblaciones rurales (valles bajos, campamentos mineros y faenas) y urbanas (pueblos y ciudades de la costa y el desierto) hablantes del castellano. Ello ha exigido a los aymara una especial preocupación por aprender el castellano. Aun para el más aislado de los pastores de la Puna, el castellano se hace indispensable para vincularse en condiciones menos injustas y odiosas con los suprasistemas económicos de los que depende. Algún aymara que no hable castellano o lo haga con deficiencias es alguien socialmente vulnerable y un candidato a la explotación por parte de otros aymara o no aymara. ${ }^{25}$

23 Al presentar estos planteamientos no queremos dejar la impresión de que concebimos los procesos que empiezan a oponer el altiplano de los valles como algo rígido y sin matices. La evolución de la lengua aymara puede ejemplificar bien la riqueza de situaciones que indudablemente existió. En efecto, en ciertos lugares, como la parte alta de la cuenca del río Aroma, el aymara se ha conservado hasta tardíamente. A la inversa, en ciertos lugares del altiplano como Parinacota, por sus íntimas relaciones con el valle de Putre, el castellano parece haberse aprendido más tempranamente que en otros sitios.

24 Sería del mayor interés para el conocimiento de la historia y sociedad aymara actual abordar las transformaciones acaecidas en la zona desde fines del siglo XVIII y, con base en sus resultados, confrontar antecedentes sociolingüísticos rescatables desde fuentes secundarias.

25 No es casualidad que el estrato socialmente más deficitario del altiplano, los peones pastores, sea en un buen número de casos bilingües incipientes respecto al castellano.
En el pasado, quizá hacia inicios del siglo XIX, cuando el aymara fue una lengua empleada de manera más generalizada por una proporción mucho mayor de población que ahora, las relaciones económicas entre aymara y no aymara no suponían el castellano para hacerse posible. Para ello había intérpretes y lenguaraces a los que cada quien recurría para hacerse entender. A medida que la región agrícola se castellaniza y que hacia el desierto y la costa fluyen importantes contingentes de población, sobre todo del centro de Chile, el aymara queda cada vez más confinado a las alturas. Se hace cada vez más una lengua de una minoría aislada. Las poblaciones aymara de este siglo, viviendo condiciones de creciente mercantilización de sus economías, de acceso cada vez más directo a los mercados urbanos, experimentando fenómenos de proletarización de migración, de inserción en economías informales, por mencionar algunas cuestiones pertinentes, se vieron obligadas a apropiarse del castellano. Circunstancias como las mencionadas suponían, para hacerse posible, un instrumento de comunicación compartido. No había precondiciones de ningún tipo para que éste pudiera ser el aymara.

Un tercer tema, por último, es el rol del Estado chileno en los cambios lingüísticos acaecidos al aymara. Como cuestión general se puede decir que el Estado ha tenido, hasta época muy reciente, una política de castellanización de los aymara. Sólo con la formación de la Corporación Nacional de Desarrollo Indígena (CONADI) y las políticas que ella impulsa se avizoran, todavía para el mediano plazo, cambios en la actitud del Estado frente a las lenguas indígenas. La política de castellanización, a su vez, debe ser entendida en el contexto histórico de una zona conquistada y anexada y dentro de un proceder más global de "chilenización" de los aymara. A ella corresponde una modalidad particularmente fuerte de integración, la asimilación irrestricta a las formas culturales y sociales características de la población del centro del país o, más bien, a la representación que se hace de lo que es o deben ser tales formas de vida.

Dos ideas recorren durante gran parte de este siglo la presencia de los organismos del Estado chileno en las zonas rurales nortinas con población aymara: la noción de progreso y la de "civilización". Ambas suponen un estado de carencia evaluado negativamente. En la primera, el supuesto es una condición 
de atraso, rusticidad, marginalidad. En la segunda, el supuesto es cultural (un estado de primitivismo o barbarie), en el que no son ajenas algunas notas de racismo. ${ }^{26}$ Según estas ideas, son poblaciones que deben dejar atrás el atavismo y vida semisilvestre para, mediante las bondades de la educación, el acceso a servicios y la vinculación con los que están en la senda del progreso, integrarse a una vida más a tono con el ser chileno. ${ }^{27}$

Una visión del mundo andino regional estructurada por conceptos del tipo descrito está lejos de otorgar a la lengua aymara algún rol en el futuro de estas poblaciones. Por el contrario, su existencia se alza como un obstáculo a una más rápida integración social y cultural. La escuela nacional obligatoria ha cumplido en este ámbito un rol destacado. Desde su formación, en la zona precordillerana primero y en el altiplano después, ha sido un eficiente instrumento de difusión de la lengua castellana. Pero, simultáneamente, ha sido también un espacio institucional en el que sistemáticamente se ha desprestigiado y reprimido el aymara como una lengua, o "dialecto" para la mayoría, inútil, perjudicial y sin valor. Roles similares, pero ciertamente con un alcance más restringido, han jugado en este sentido las dotaciones policiales asentadas en la zona y la conscripción militar. La presencia del Estado en las zonas interiores se ha hecho cada vez más efectiva y diversificada (creación de municipios rurales, instalación de algunos servicios, presencia frecuente de otros). Ya sea que tengan un rol pasivo o activo en la castellanización y en la desaymarización de las zonas interiores, el Estado a través de sus diversos organismos ha sido un factor de primera importancia en la dinámica del cambio lingüístico andino.

\begin{tabular}{|l|c|c|c|c|}
\hline \multicolumn{1}{|c|}{ Sectores y subsectores geográficos } & $\begin{array}{c}\text { Población } \\
\text { aymara }\end{array}$ & $\begin{array}{c}\text { \% sobre total de } \\
\text { población (*) }\end{array}$ & $\begin{array}{c}\text { Conocen la } \\
\text { lengua aymara }\end{array}$ & $\begin{array}{c}\text { \% de la } \\
\text { población } \\
\text { aymara }\end{array}$ \\
\hline Altiplano & 3188 & $100 \%$ & 3089 & $96.9 \%$ \\
* Altiplano norte & 1458 & $100 \%$ & 1413 & $96.9 \%$ \\
* Altiplano sur & 1730 & $100 \%$ & 1676 & $96.9 \%$ \\
\hline Valles, quebradas y oasis & 10358 & $709 \%$ & 5835 & $56.3 \%$ \\
* Precordillera norte & 1901 & $913 \%$ & 1072 & $56.4 \%$ \\
* Precordillera sur & 4141 & $778 \%$ & 2335 & $56.4 \%$ \\
* Valles bajos & 4216 & $585 \%$ & 2378 & $56.4 \%$ \\
* Provincia de El Loa rural & $100(?)$ & - & 50 & $50.0 \%$ \\
\hline Totales rurales & 13546 & $757 \%(* *)$ & 8924 & $65.9 \%$ \\
\hline Centros urbanos & 20000 & $79 \%$ & 6000 & $30.0 \%$ \\
\hline Totales generales & 33546 & $122 \%$ & 14924 & $44.5 \%$ \\
\hline
\end{tabular}

Fuente: Censo de población de 1982

Encuesta sociolingüística (1985)

(*) En el caso rural se refiere al total de la población agrícola y en el de los centros urbanos a la población de las ciudades de Arica, Iquique y Pozo Almonte, tomando como base los resultados del Censo de Población de 1982

(**) Referido sólo a la población agrícola de la I Región.

Cuadro 1. Población aymara y número y porcentaje de individuos con conocimientos de la lengua aymara según sectores y subsectores geográficos (Gundermann 1994).

26 Por ejemplo, Alfredo Wormald Cruz, un profesor universitario relacionado al Plan Andino de la Junta de Adelanto de Arica, quien podría ser calificado como un buen expositor de ciertos conceptos altamente estereotipados que durante las décadas de los 60 y 70 mantenían diversos funcionarios públicos relacionados con la zona interior y que, de algún modo, también hacían suyos los programas que impulsaban (Wormald s/f).
27 Planteamos aquí ideas muy amplias de un problema de la mayor importancia para la comprensión de la historia de los aymara durante el período chileno: el rol que le ha tocado jugar al Estado chileno en los profundos cambios que sacuden a esta minoría étnica. 


\section{HANS GUNDERMANN}

\section{REFERENCIAS CITADAS}

BOLLAERT, W., 1975 [1860]. Descripción de la Provincia de Tarapacá. Norte Grande 1(3-4): 459-479.

GONZALEZ, S., 1990. El aymara de la Provincia de Iquique - Chile y la educación nacional. Cuadernos de Educación Intercultural 1, Taller de Estudios Regionales, Iquique.
GUNDERM ANN, H., 1994. ¿Cuántos hablan en Chile la lengua aymara? Revista de Lingüística teórica y aplicada 32: 125-139.

WORMALD, A., s/f. El mestizo en el departamento de Arica. Ediciones Ráfaga, Santiago. 